\title{
Increased plasma viscosity and erythrocyte aggregation: indicators of an unfavourable clinical outcome in patients with unstable angina pectoris
}

Franz-Josef Neumann, Hugo A Katus, Eike Hoberg, Peter Roebruck, Martin Braun, Heide-Marlen Haupt, Harald Tillmanns, Wolfgang Kübler

\begin{abstract}
Objective-To determine the prognostic significance of altered plasma viscosity and erythrocyte aggregation in unstable angina.

Design-A prospective study of 96 consecutive patients with unstable angina allocated to one of two groups according to predefined threshold values for plasma viscosity and erythrocyte aggregation at study entry. The patients received a standardised treatment and were followed up for six months or until angioplasty or bypass surgery.

Main outcome measure-Frequency of myocardial infarction.
\end{abstract}

Results-Myocardial infarctions occurred in $7 / 26$ patients with a plasma viscosity $\geqslant 1.38 \mathrm{mPa}$ and in $8 / 35$ with a rate constant of erythrocyte aggregate formation $\geqslant 0.5 \mathrm{mPa}$ (corrected for plasma viscosity) but in only $4 / 70$ with a plasma viscosity $<1.38 \mathrm{mPa} s$ and in $3 / 61$ with an erythrocyte aggregation $<0.5 \mathrm{mPa}$ (odds ratios: $6.1 \quad(95 \%$ confidence interval 1.3 to 31 ), $p=0.008$, and $5.7(95 \%$ CI 1.2 to 35$), p=0.016)$. Plasma viscosity and erythrocyte aggregation were more predictive of myocardial infarction than age, male gender, fibrinogen concentration, ST segment abnormalities, or coronary score. Furthermore, Holter monitoring with ST segment analysis showed that ischaemic episodes were more common in patients in whom the rate constant of erythrocyte aggregate formation was $>0.5 \mathrm{mPa}(15 / 27$ v $17 / 50, \quad \mathrm{p}=0.029)$. Cardiac troponin $T$ release was increased in patients with a plasma viscosity of $>1.38 \mathrm{mPa}$ s $(10 / 26 v 9 / 70$, $\mathbf{p}=\mathbf{0 . 0 1 0}$ ).

Conclusions-In patients with unstable angina a considerable increase in plasma viscosity and erythrocyte aggregation identified a subgroup of patients at a high risk of acute myocardial infarction in whom medical treatment was likely to be unsuccessful.

The fluid-mechanical properties of the blood are major determinants of microcirculatory perfusion ${ }^{1-3}$ and oxygen transport. ${ }^{4}$ In dogs a rise in blood viscosity decreased the capillary perfusion of post-stenotic areas of the coron- ary circulation. ${ }^{5}$ Furthermore, in humans alterations of blood fluidity interfered with coronary vasodilator capacity. ${ }^{67}$ Haemorrheological abnormalities in ischaemic heart disease $^{8-13}$ may, therefore, adversely affect the course of the disease. There is no direct evidence to support this hypothesis, however.

In a recent study of haemorrheological abnormalities in coronary artery disease a considerable increase in plasma viscosity and erythrocyte aggregation was found in patients with unstable angina. ${ }^{14}$ This association could not be attributed to changes in the risk factor profile or the angiographic severity of coronary artery disease. ${ }^{14}$ The increase in plasma viscosity and erythrocyte aggregation in unstable angina may, therefore, be related directly to the course of ischaemic heart disease. In the present study we investigated the prognostic significance of increased plasma viscosity and erythrocyte aggregation in patients with unstable angina.

\section{Patients and methods \\ PATIENT SELECTION}

We studied 120 consecutive patients admitted to the department of cardiology for treatment of unstable angina. Unstable angina was defined by the presence of at least one of the following symptoms: (a) angina of recent onset-that is within six weeks or less-brought on by minimal exertion; $(b)$ crescendo angina-that is, more severe, prolonged, or more frequent anginal attacks, superimposed on chronic effort angina; $(c)$ angina at rest lasting for at least 15 minutes. Exclusion criteria were $(a)$ interfering non-cardiac diseases-that is, anaemia, infection, tumours, connective tissue diseases, diabetes mellitus, thyrotoxicosis; $(b)$ cardiac diseases other than coronary artery diseasefor example, dilated or hypertrophic cardiomyopathy, valvar heart disease (except for minor mitral regurgitation); (c) left bundle branch block; $(d)$ previous coronary artery bypass surgery or percutaneous transluminal coronary angioplasty; $(e)$ myocardial infarction less than four months before study entry; $(f)$ contraindications for coronary angiography or standardised antianginal treatment; $(g)$ absence of significant coronary artery disease-stenoses with $<50 \%$ narrowing in luminal diameter; $(h)$ evolving myocardial infarction or intervention by coronary angioplasty or bypass surgery less than eight hours after study entry; $(i)$ deviation from the study protocol. The last three criteria 
led to 24 patients being excluded retrospectively: 13 patients with coronary artery narrowing $<50 \%$, six with an early myocardial infarction, and five because of a deviation from the study protocol. These deviations were withdrawal of the standardised medical treatment because of side effects in two patients, premature balloon angioplasty in two patients, and only non-invasive investigation in one patient. Ninety six patients remained within the study ( 84 men and 12 women; median age 58 (upper quartile 53.5 and lower quartile 65)).

\section{STUDY PROTOCOL}

Eligible patients were recruited within four hours after admission to hospital. Blood was drawn for haemorrheological and marker protein analysis immediately after study entry. During the first 48 hours treatment was primarily conservative-that is standardised antianginal treatment with: aspirin $(500 \mathrm{mg}$ / day); heparin ( $200 \mathrm{IU} / \mathrm{kg}$ body weight per day); metoprolol (dosage according to resting pulse rate, therapeutic aim: $50-55$ beats per minute); nitrates, initially intravenous (dosage according to systolic blood pressure, therapeutic aim: $100-120 \mathrm{~mm} \mathrm{Hg}$ ), with isosorbide mononitrate (20 mg twice a day) after suppression of resting angina for at least 24 hours; and nifedipine, if control of blood pressure was insufficient. Coronary angioplasty or bypass surgery were performed as an early intervention for refractory angina, increasing ST segment changes on a 12 lead electrocardiogram, and signs of evolving myocardial infarction. All patients were followed up either for six months or until intervention. The attending cardiologist decided on invasive intervention and its timing.

During the first $\mathbf{4 8}$ hours Holter monitoring was performed for ST segment analysis and the patient was asked to keep a detailed record of any symptoms. A 12 lead electrocardiogram was recorded at admission to hospital, eight hours after admission to hospital, and once daily thereafter for the first week. The serum activities of creatine kinase and its $\mathrm{MB}$ isoenzyme ${ }^{15}$ and the plasma concentration of cardiac troponin $\mathrm{T}$ (see below) were measured every eight hours for the first 24 hours, every 12 hours for the next $\mathbf{4 8}$ hours, and once daily thereafter for the first week. During episodes of severe angina in hospital additional blood samples were taken to measure routinely available cardiac marker proteins and a 12 lead electrocardiogram was recorded.

The study was approved by our ethics committee. All patients and their attending physicians gave their informed consent after a thorough explanation of the study protocol and its potential hazardous and beneficial effects. If the attending physician decided to deviate from the study protocol the patient was excluded from the study.

\section{HAEMORRHEOLOGICAL VARIABLES}

Blood was taken without a tourniquet from the antecubital vein with EDTA as anticoagulant. Plasma viscosity was determined by capillary viscometry on a Coulter-Harkness viscometer. ${ }^{16}$ Erythrocyte aggregation was analysed photometrically at an adjusted haematocrit of $45(1) \%$ on a previously described aggregometer (Myrenne $\mathrm{GmbH}$, Roetgen, Federal Republic of Germany). ${ }^{17} 18$ The rate constant of erythrocyte aggregate formation in stasis was determined by the increase in photovoltage during the first eight seconds after mixing. As recommended elsewhere ${ }^{18}$ the rate constant of erythrocyte aggregate formation was multiplied by plasma viscosity to correct for the damping of aggregate formation by plasma viscosity.

The study group was divided into subgroups according to prospectively defined threshold values for the haemorrheological variables measured at study entry. The threshold values were $\geqslant 1.38 \mathrm{mPa}$ s for plasma viscosity and $\geqslant 0.5 \mathrm{mPa}$ for the corrected rate constant of erythrocyte aggregate formation. These threshold values represented the upper terciles of the distribution of these variables for patients with unstable angina in a previous study on haemorrheological abnormalities in coronary artery disease. ${ }^{14}$

\section{CO-VARIABLES}

We analysed the prognostic significance of the patient's age and sex and of the plasma fibrinogen concentration and the ST segment abnormalities on the 12 lead electrocardiogram at admission to hospital. Plasma fibrinogen concentrations were measured by Clauss's method. ${ }^{19}$ Patients were assumed to have ST segment abnormalities if they had horizontal or downsloping ST segment depression of at least $0.1 \mathrm{mV} 0.08 \mathrm{~s}$ after the J point in at least one limb lead or two precordial leads. $T$ wave abnormalities were defined as biphasic $T$ waves in any lead, or a negative $T$ wave in leads V2$\mathrm{V} 6$, or a deviation in the horizontal $\mathrm{T}$ wave axis of $>30^{\circ}$ from the QRS axis.

Coronary angiography was performed by Judkins technique ${ }^{20}$ and the angiograms were evaluated as described earlier. ${ }^{4}$ The coronary artery lesions were graded as luminal reduction visually estimated by two independent cardiologists who were unaware of the test results. The lesions were scored as follows: grade 1 , $<25 \%-50 \%$; grade $2,>50 \%-75 \%$; grade 3 , $>75 \%-90 \%$; grade 4 , lumen almost totally obliterated but still anterograde flow; grade 5, occlusion. The total coronary score was the sum of the scores of each lesion.

\section{MAJOR END POINTS}

The following were major end points: non- $Q$ wave myocardial infarction, evolving $Q$ wave myocardial infarction, and cardiac death. A non- $Q$ wave myocardial infarction was defined as a rise in serum creatine kinase activity of $>100 \mathrm{U} / 1$ with a concomitant rise in $\mathrm{MB}$ isoenzyme to at least $8 \%$ of the total creatine kinase activity, together with newly developed significant ST segment depression or $\mathrm{T}$ wave inversion (see above), and/or a prolonged $(\geqslant 15$ minutes) episode of angina at rest. We assumed that a $Q$ wave myocardial infarction was evolving if the sum of the ST segment elevation that lasted $\geqslant 15$ minutes exceeded $0.3 \mathrm{mV}$. The diagnosis of an evolving myocardial infarction 

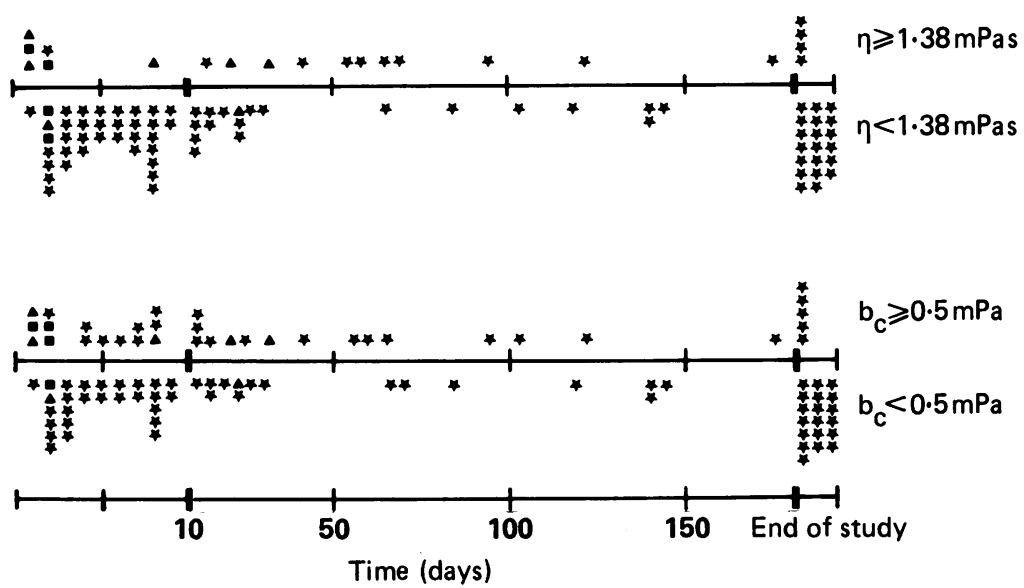

Figure 1 Distribution of cardiac events and censored observation times over the study period for the groups defined by plasma viscosity and erythrocyte aggregation. All exclusions before $(*)$ end of study (182 days) were due to interventions. $\eta$, plasma viscosity; $b_{c}$, corrected rate constant of erythrocyte aggregate formation; $(\Delta)$, non- $Q$ wave myocardial infarction; ( $Q$ ) $Q$ wave myocardial infarction.

prompted immediate systemic thrombolysis or coronary angioplasty.

\section{MINOR END POINTS}

Minor end points were features that indicated the persistence of unstable angina despite maximal medical treatment: (a) recurrent episodes of angina at rest; $(b)$ progression of ST segment abnormalities on the 12 lead electrocardiogram, (c) episodes of ischaemia on Holter monitoring, $(d)$ a significant increase in the serum concentration of cardiac troponin $T$. The minor end points were evaluated between 16 hours and 48 hours after initiation of the standard medical treatment.

ST segment abnormalities were assumed to be progressive if the sum of the ST segment depressions $0.08 \mathrm{~s}$ after the $\mathrm{J}$ point rose by at least $0.2 \mathrm{mV}$. Frequency modulated Holter recordings (Oxford MR 20) were monitored on leads CC5 and CM5 and evaluated as described earlier. ${ }^{21}$ Adequate recordings were obtained from 77 patients. Episodes of horizontal or downsloping ST segment depressions $\geqslant 0.1 \mathrm{mV}$ or of ST segment elevations $\geqslant 0.1 \mathrm{mV}$ were classified as ischaemic if they lasted for at least $60 \mathrm{~s}$. The serum immunoreactivity of cardiac troponin $T$ was analysed by a newly developed enzyme linked immunoassay. ${ }^{22} 23$ When serum concentrations of cardiac troponin $\mathrm{T}$ exceeded $0.5 \mathrm{mg} / \mathrm{dl}$ on two occasions the increase was regarded as significant.

Table 1 Baseline characteristics (median (quartiles) or numbers (percentages)) of the study population in the two subgroups defined by plasma viscosity

\begin{tabular}{|c|c|c|c|}
\hline \multirow[b]{2}{*}{ Characteristics } & \multicolumn{3}{|l|}{ Plasma viscosity } \\
\hline & $\geqslant 1.38 \mathrm{mPa} \mathrm{s}$ & $<1.38 \mathrm{mPas}$ & $p^{\star}$ \\
\hline $\begin{array}{l}\text { Age } \\
\text { Male } \\
\text { Fibrinogen }(\mathrm{g} / \mathrm{l}) \\
\text { ST abnormalities } \\
\text { T abnormalities } \\
\text { High grade stenoses: } \\
90 \% \text { luminal reduction } \\
\text { " } 99 \% \text { " luminal reductiont } \\
\text { Coronary score }\end{array}$ & $\begin{array}{c}59(53,64) \\
23 / 26(88 \%) \\
345(291,374) \\
11 / 26(42 \%) \\
16 / 26(62 \%)\end{array}$ & $\begin{array}{c}59(54,66) \\
59 / 70(84 \%) \\
285(250,321) \\
19 / 70(27 \%) \\
46 / 70(66 \%)\end{array}$ & $\begin{array}{l}0.86 \\
0.75 \\
0.004 \\
0.22 \\
0.65 \\
1.00\end{array}$ \\
\hline
\end{tabular}

$\star T$ wo-tailed exact test in contingency tables and $W$ ilcoxon test for continuous variables. tA coronary stenosis with an almost totally obliterated lumen but still with anterograde flow.
STATISTICAL ANALYSIS

To compare the two groups we tested frequencies by Fisher's exact test and continuous variables by the Mann-Whitney-Wilcoxon rank sum test. Continuous variables were expressed as medians and lower and upper quartiles. We used Kaplan-Meier analyses to estimate event free survival. The statistical significance of odds ratios for major end points was assessed by Fisher's exact test and their corresponding exact $95 \%$ confidence intervals were calculated. Because Fisher's exact test does not take account of different observation times, we also calculated log rank tests of the event free times to strengthen the evidence from previous analysis. Because of the low number of events, however, $p$ values of the log rank test can only be regarded as approximate. For the two tailed test $p>0.05$ was regarded as not significant. The results of Cox model analyses are not reported in the present study because the numbers of events were too small. For the same reason we did not fit models with two or more factors. We used the SPSS and the $S A S$ statistical packages for analysis.

\section{Results}

CHARACTERISTICS OF THE STUDY POPULATION

Sixty eight of the 96 patients in our study underwent coronary angiography within 24 hours of study entry and in the remaining 28 patients coronary angiography was performed at a median of 49 hours after study entry. Seven non- $Q$ wave myocardial infarctions occurred and four patients had an evolving $Q$ wave myocardial infarction. Despite immediate intervention all patients with evolving $Q$ wave myocardial infarction showed a significant rise in total serum creatine kinase and $\mathrm{MB}$ isoenzyme activity. One patient died from cardiogenic shock caused by extensive acute anterior myocardial infarction. Thirty eight patients had coronary angioplasty and 23 had coronary bypass surgery. The median observation time in the entire study group was 15 days (quartiles: 5, 178.5) (fig 1). All cardiac events occurred within the 33 days after study entry (median three days) (quartiles: $1 \cdot 4,21$ ) (fig 1).

Twenty six patients had a plasma viscosity $\geqslant 1.38 \mathrm{mPa} \mathrm{s}$ and 35 had a corrected rate constant of erythrocyte aggregate formation $\geqslant 0.5 \mathrm{mPa}$. In 20 patients both haemorrheological variables were above the prospectively defined threshold value. The two subgroups defined by plasma viscosity (table 1 ) and the two subgroups defined by the corrected rate constant of erythrocyte aggregate formation (table 2) were not significantly different in terms of age, sex distribution, coronary score, the frequencies of high grade coronary stenoses, or of ST segment or $T$ wave abnormalities at study entry. Plasma fibrinogen concentrations, however, were significantly higher in the subgroups with plasma viscosity or corrected rate constant of erythrocyte aggregate formation above the threshold value than in those with haemorrheological variables below the threshold values. Observation times in the subgroups with plasma viscosity or corrected rate 
Table 2 Baseline characteristics (median (quartiles) or numbers (percentages)) of the study population in the two subgroups defined by erythrocyte aggregation

\begin{tabular}{lccl}
\hline \multicolumn{4}{l}{ Erythrocyte aggregation } \\
\cline { 2 - 4 } Characteristic & $b_{c} \geqslant 0.5 \mathrm{mPa}$ & $b_{c}<0.5 \mathrm{mPa}$ & $p$ \\
\hline Age & $60(57,66)$ & $58(51,65)$ & 0.79 \\
Male & $32 / 35(91 \%)$ & $50 / 61(82 \%)$ & 0.25 \\
Fibrinogen (g/1) & $343(289,381)$ & $284(243,314)$ & 0.002 \\
ST abnormalities & $10 / 35(29 \%)$ & $33 / 61(33 \%)$ & 0.82 \\
Tabnormalities & $20 / 35(57 \%)$ & $42 / 61(69 \%)$ & 0.29 \\
High grade stenoses: & $16 / 35(46 \%)$ & $21 / 61(34 \%)$ & 0.43 \\
90\% & $6 / 35(17 \%)$ & $9 / 61(15 \%)$ & \\
"99\%" & $8.0(6.0,14 \cdot 0)$ & $7 \cdot 0(4 \cdot 0,12 \cdot 0)$ & $0 \cdot 26$ \\
\hline
\end{tabular}

$b_{c}$, corrected rate constant of erythrocyte aggregate formation. See footnote to table 1.

Table 3 Frequencies of minor and major end points in the two subgroups defined by plasma viscosity

\begin{tabular}{|c|c|c|c|}
\hline \multirow[b]{2}{*}{ End point } & \multicolumn{3}{|c|}{ Plasma viscosity } \\
\hline & $\begin{array}{l}\geqslant 1.38 \mathrm{mPas} \\
(\mathrm{n}(\%))\end{array}$ & $\begin{array}{l}<1.38 \mathrm{mPas} \\
(\mathrm{n}(\%))\end{array}$ & $p^{\star}$ \\
\hline \multicolumn{4}{|l|}{ Minor end points: } \\
\hline 12 lead ECG & $5 / 26(19)$ & $11 / 70(16)$ & 0.76 \\
\hline Holter monitoring & $12 / 21(57)$ & $20 / 56(36)$ & $0 \cdot 12$ \\
\hline Troponin $\mathrm{T}$ & $10 / 26(38)$ & $9 / 70(13)$ & 0.010 \\
\hline Angina at rest & $12 / 26(46)$ & $37 / 70(53)$ & 0.65 \\
\hline \multicolumn{4}{|l|}{ Major end points: } \\
\hline Non-Q wave $M I$ & $5 / 26(19)$ & $2 / 70$ & \\
\hline $\mathrm{Q}$ wave $\mathrm{MI}$ & $2 / 26(8)$ & $2 / 70$ (3) & \\
\hline
\end{tabular}

constant of erythrocyte aggregate formation above the threshold value were shorter than those in the rest of the patients (median (quartiles): 11.5 days $(5,94) v 17.5$ days $(5,182)$ for plasma viscosity and 13 days $(5,194) v 19$ days $(5,182)$ for the corrected rate constant of erythrocyte aggregate formation (fig 1 ).

\section{MINOR END POINTS}

In patients with a plasma viscosity $\geqslant 1.38 \mathrm{mPa}$ s continued release of troponin $\mathrm{T}$ was more common than in patients with a plasma viscosity below the threshold value (table 3). Furthermore, the subgroup with raised plasma viscosity tended to have more episodes of persistent ischaemia on Holter monitoring (table 3).

In patients with a corrected rate constant of erythrocyte aggregate formation of $\geqslant 0.5 \mathrm{mPa}$, persistent ischaemic episodes on Holter monitoring and progression of ST segment abnormalities on the 12 lead electrocardiogram despite maximal medical treatment were significantly more frequent than in the subgroup with a corrected rate constant of erythrocyte aggregate formation $<0.5 \mathrm{mPa}$ (table 4). A trend towards a higher frequency of troponin $\mathrm{T}$

Table 4 Frequencies of minor and major end points in the two subgroups defined by erythrocyte aggregation

\begin{tabular}{llll}
\hline & \multicolumn{3}{l}{ Erythrocyte aggregation } \\
\cline { 2 - 4 } $\begin{array}{l}b_{c} \geqslant 0.5 \mathrm{mPa} \\
(n(\%))\end{array}$ & $\begin{array}{l}b_{c}<0.5 \mathrm{mPa} \\
(n(\%))\end{array}$ & $p^{\star}$ \\
\hline End point & & & \\
\hline Minor end points: & $10 / 35(29)$ & $6 / 61(10)$ & 0.024 \\
12 lead ECG & $15 / 27(56)$ & $17 / 50(34)$ & 0.029 \\
Holter monitoring & $10 / 35(29)$ & $9 / 61(15)$ & 0.12 \\
Troponin T & $17 / 35(49)$ & $32 / 61(52)$ & 0.75 \\
Angina at rest & $5 / 35(14)$ & $2 / 61(3)$ & 0.029 \\
Major end points: & $3 / 35(9)$ & $1 / 61(2)$ & \\
Non-Q wave MI & & & \\
Q wave MI & &
\end{tabular}

$b_{c}$, corrected rate constant of erythrocyte aggregate formation. ${ }^{\star} T$ wo tailed $p$ values for exact test. release was also found in patients with increased erythrocyte aggregation. The frequency of recurrent episodes of angina at rest was not related to any of the haemorrheological variables investigated.

\section{MAJOR END POINTS}

Cardiac events, particularly non-Q wave myocardial infarctions, were significantly more common in those with a higher plasma viscosity (table 3 ) or a higher corrected rate constant of erythrocyte aggregate formation (table 4). Table 5 shows the corresponding odds ratios. Kaplan-Meier lifetable analyses (figs 2 and 3) showed that patients with haemorrheological variables above the threshold values had significantly lower event free survival rates than patients with haemorrheological variables below the threshold values.

Analysis of the rate constant of erythrocyte aggregate formation without correction for plasma viscosity yielded similar results as the corrected rate constant of erythrocyte aggregate formation. Dichotomisation by the upper tercile showed that seven of 32 patients with an uncorrected rate constant of erythrocyte aggregation $\geqslant 0.38 \mathrm{~s}^{-1}$ had a myocardial infarction, whereas this occurred in only four of the 64 patients with an uncorrected rate constant of erythrocyte aggregation below the upper tercile (odds ratio: 3.5 (95\% CI 0.96 to $36)$, exact $p=0.038, \log \operatorname{rank} p=0.023)$.

ANALYSIS OF CO-VARIABLES WITH RESPECT TO THE RISK OF A CARDIAC EVENT

All co-variables analysed were less predictive of the risk of a cardiac event than the haemorrheological variables (table 5). Only an age above the median was associated with a significantly increased relative risk in the log rank test, but not in Fisher's exact test. Similarly, ST segment abnormalities at study entry and a plasma fibrinogen concentration above the upper tercile tended to increase the risk of a cardiac event.

\section{Discussion}

The present study is the first to show a relation between impaired blood flow properties and an unfavourable clinical course of coronary artery disease. In patients with unstable angina a plasma viscosity $\geqslant 1.38 \mathrm{mPa}$ or a corrected rate constant of erythrocyte aggregate formation of $\geqslant 0.5 \mathrm{mPa}$ on admission to hospital the risk of developing an acute myocardial infarction within 33 days was increased fivefold and medical treatment was less likely to be successful. In these subgroups medical treatment was less likely to prevent the progression of ST segment abnormalities and/or to suppress recurrent episodes of ischaemia.

\section{HAEMORRHEOLOGICAL VARIABLES AND} CO-VARIABLES

In an earlier study we found that plasma viscosity and erythrocyte aggregation were the variables most closely related to the clinical manifestation of coronary artery disease. ${ }^{14} \mathrm{We}$ used the corrected rate constant of erythrocyte 
Table 5 Odds ratios for cardiac events

\begin{tabular}{lllll}
\hline Variable & Odds ratio $(95 \%$ CI $)$ & Observation times ${ }^{\star}$ & Exact $p^{\dagger}$ & Log rank $^{\ddagger}$ \\
\hline Plasma viscosity & $6.1(1.34$ to 31$)$ & $17.5 v 11.5$ & 0.008 & 0.004 \\
Erythrocyte aggregation $\$$ & $5.7(1.23$ to 35$)$ & $19.0 v 13.0$ & 0.016 & 0.009 \\
Age & $5.1(0.95$ to 50$)$ & $8.0 v 21.0$ & 0.051 & 0.043 \\
Female & $0.0(0.00$ to 2.3$)$ & $5.5 v 23.0$ & 0.36 & 0.22 \\
Fibrinogen & $2.6(0.59$ to 12$)$ & $11.0 v 19.0$ & 0.18 & 0.13 \\
ECG & $3.1(0.69$ to 14$)$ & $15.0 v 25.0$ & 0.092 & 0.098 \\
Stenosis $\geq 90 \%$ & $1.0(0.24$ to 4.6$)$ & $76.0 v 8.5$ & 1.00 & 0.77 \\
Coronary score & $0.82(0.16$ to 3.5$)$ & $11.0 v 42.0$ & 0.65 & 0.65 \\
\hline
\end{tabular}

*Median observation time of the subgroup with the variable below the cut off point compared with that of the subgroup with the respective variable above the cut off point; †Level of significance in two tailed Fisher's exact test; $\$$ Level of significance in the two tailed log rank test; \$Rate constant of red cell aggregate formation. Age, age above median; fibrinogen, fibrinogen concentration above the upper tercile; ECG, ST segment abnormalities on 12 lead ECG at study entry; stenosis $\geqslant 90 \%$, patients with high grade stenoses (" $90 \%$ " and "99\%") exclusive of occlusions; coronary score, coronary score above median.

aggregate formation to characterise erythrocyte aggregation because it is least affected by shear induced artefacts and phase separation. It has been suggested that the corrected rate constant of erythrocyte aggregate formation reflects the strengths of the protein bonds between aggregating erythrocytes. ${ }^{18}$

The two haemorrheological variables that we studied had a similar predictive value. There was a considerable overlap between the subgroups defined by erythrocyte aggregation or plasma viscosity. The similarity between the two haemorrheological variables studied cannot be attributed simply to the correction of the rate constant of erythrocyte aggregation for plasma viscosity, because analysis of this variable with and without correction for plasma viscosity gave similar results. The similarity between the results for plasma viscosity and erythrocyte aggregation, however, may be explained by the fact that both variables are determined by plasma protein composition. ${ }^{24}$ The subgroup with the increased plasma viscosity was slightly smaller than that with an increase in erythrocyte aggregation. Accordingly, the threshold value for plasma viscosity seemed to be more discriminative for the end points that were less common-such as the major end points and the minor end point of troponin $T$ release. On the other hand, the more common end points-such as persistence

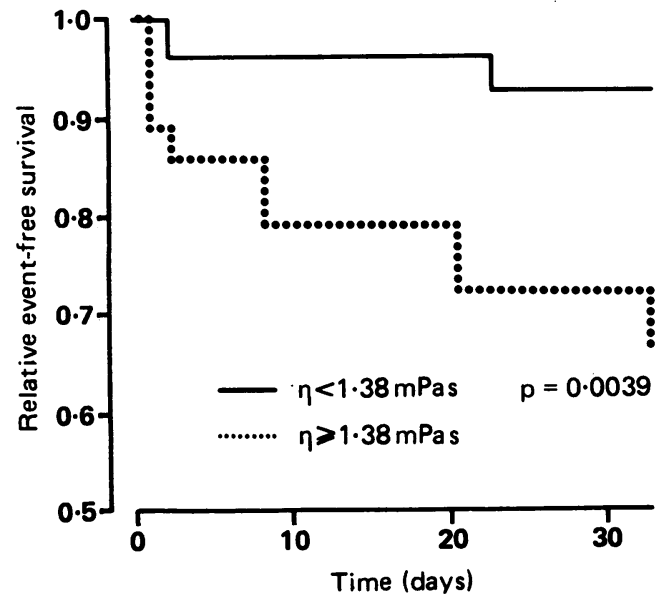

Figure 2 Kaplan-Meier survival distribution functions for patients with a plasma viscosity $\geqslant 1.38 \mathrm{mPa} s$ for patients with a plasma viscosity $\geqslant 1.38 \mathrm{mPa}$
(broken line) and for patients with a plasma viscosity $<1.38 \mathrm{mPa}$ ( (solid line). Survival distribution functions give estimates of the relative number of event free patients as a function of time. Cardiac events were $Q$ wave myocardial infarction, non- $O$ wave myocardial

infarction, and cardiac death. $\eta$, plasma viscosity; $p$, twotailed level of significance for the difference in event free survival between the two subgroups in the log rank test. of ischaemic episodes on Holter monitoringseemed to be better predicted by erythrocyte aggregation.

Comparison of the relative prognostic value of the haemorrheological variables and the covariables in multifactorial models, such as Cox models, was hampered by the small number of cardiac events in the present study. So we cannot exclude the possibility that some of the predictive power of the haemorrheological variables was really due to the co-variation of age, sex, ST segment abnormalities at study entry, plasma fibrinogen concentration, prevalence of high grade stenoses, and coronary score. Except for plasma fibrinogen concentrations, however, the subgroups defined by plasma viscosity or erythrocyte aggregation did not differ significantly in terms of any of the covariables analysed. Furthermore, in the univariate analysis of the risk of a cardiac event plasma viscosity and erythrocyte aggregation were substantially more predictive than the covariables analysed. In particular, plasma fibrinogen was not of significant prognostic value. We conclude that plasma viscosity or erythrocyte aggregation provided substantial additional information about the risk of myocardial infarction in unstable angina.

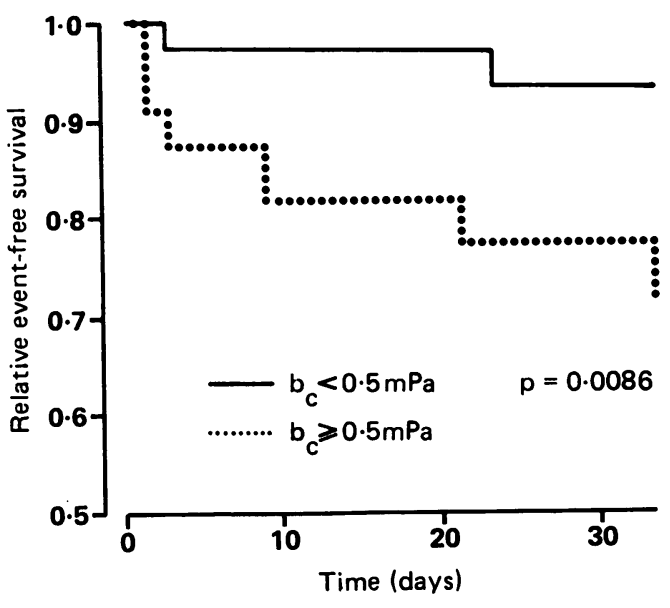

Figure 3 Kaplan-Meier survival distribution functions for patients with a corrected rate constant of erythrocyte aggregate formation $\geqslant 0.5 \mathrm{mPa}$ (broken line) and for patients with a corrected rate constant of erythrocyte aggregate formation $<0.5 \mathrm{mPa}$ (solid line). Survival distribution functions give estimates of the relative number of event-free patients as a function of time. $Q$ number of event-free patients as a function of time. $Q$ infarction, and cardiac death were taken as cardiac events. $b$, corrected rate constant of erythrocyte aggregate formation; $p$, two-tailed level of significance for the difference in event free survival between the two subgroups in the log rank test. 


\section{METHODOLOGICAL CONSIDERATIONS}

Because of the study protocol and ethical constraints our results may have been biased by differences in medical treatment and in revascularisation by angioplasty or bypass surgery. Even though care was taken to apply a standard medical treatment we cannot assume that medical treatment was identical in all patients. The differences in medical treatment, however, were not influenced by differences in the haemorrheological variables because the attending cardiologist was unaware of them. This excludes a substantial bias from differences in medical treatment.

The patients were not followed up beyond revascularising procedures, because any further cardiac event might have been related to the procedure performed. The frequencies of cardiac events depend on observation times and thus on the timing of the intervention. For ethical reasons the decision for early intervention had to be left to the attending cardiologist. Patients who were judged to have more severe disease probably had earlier intervention than the rest of the patients. This might bias the frequencies of cardiac events in the subgroups defined by plasma viscosity and erythrocyte aggregation, if the severity of the disease was related to the haemorrheological variables. To exclude potential bias from different observation times we analysed the event free observation times by Kaplan-Meier estimators and log rank tests. According to these analyses the levels of significance for the differences in event free survival between the groups defined by plasma viscosity and erythrocyte aggregation were substantially higher than those simply calculated from the frequencies of cardiac events. This can be attributed to the fact that the observation times in the groups with plasma viscosity and erythrocyte aggregation above the threshold values were even shorter than in those with the lower values for haemorrheological variables. Hence, bias from different observation times caused some underestimation of the cardiac risk in patients in whom haemorrheological variables were increased.

\section{PATHOPHYSIOLOGICAL AND CLINICAL IMPLICATIONS}

The present study showed a close relation between an increased plasma viscosity or erythrocyte aggregation and the early outcome in unstable angina. The results, however, do not prove that the haemorrheological changes themselves adversely affect the clinical outcome. There is increasing evidence that ischaemia can induce an acute phase response with an increase in erythrocyte aggregation and plasma viscosity even before clinically overt necroses occur. ${ }^{14}{ }^{25-28}$ In ischaemic heart disease acute phase reactants may serve as markers of an advanced disease which in itself may carry more serious prognosis. Among the acute phase reactants, however, those capable of affecting the course of the disease may be assumed to be most closely related to prognosis. This may explain why plasma viscosity and erythrocyte aggregation are more predictive of the cardiac risk than fibrinogen, one of the classic acute phase reactants..$^{25}$ In clinical practice plasma viscosity and erythrocyte aggregation may help to identify subgroups of patients with unstable angina who are at increased risk of an early unfavourable clinical outcome.

This study was supported by a grant from the Wilhelm SanderStiftung, Munich, Germany.

1 Dormandy JA. Influence of blood viscosity on blood flow and the effect of low molecular weight dextran. $B M J 1971$; iv:716-9.

2 Wells R. Microcirculation and coronary blood flow. Am J Cardiol 1972;29:847-50.

3 Chien S. Hemorheology in clinical medicine. Clin Hemorheol 1982;2:137-42.

4 Finch CA, Lenfant C. Oxygen transport in man. $N$ Engl $J$ Med 1972;286:407-9.

5 Most AS, Ruocco NA, Gewirtz H. Effect of a reduction in blood viscosity on maximal myocardial oxygen delivery distal to a moderate coronary stenosis. Circulation 1986; distal to a mod

6 Volger E, Strauer BE. Hemorheological abnormalities as a possible cause of disturbed coronary hemodynamics in patients with normal coronary angiogram. Biorheology 1981;18:109-17.

7 Strauer BE. Rheologische Ursachen der Koronarinsuffizienz. Dtsch Med Wochenschr 1981;106:1490-2.

8 Nicolaides AM, Bowers R, Horobourne T, Kidner PM, Bestsman EM. Blood viscosity, red cell flexibility, haematocrit and plasma fibrinogen in patients with angina. Lancet 1977;ii:943-5.

9 Lowe GDO, Drummond MM, Lorimer AR, et al. Relation between extent of coronary artery disease and blood viscosity. $B M J 1979 ; \mathrm{i}: 673-4$.

10 Boss $M$, Wietelmann $\mathrm{H}$, Bierner $\mathrm{M}$, et al. Red blood cell aggregation in men with coronary artery disease. Eur $J$ Cardiol 1980;12:47-53.

11 Fuchs J, Weinberger I, Rotenberg Z, et al. Plasma viscosity in ischemic heart disease. Am Heart J 1984;108:435-9.

12 Low J, Dodds AJ, McGrawth M, Biggs JC. Red cell deformability and other haemorheologic variables in stable coronary artery disease. Thromb Res 1985;38: 269-74.

13 Rainer CR, Kawanishi DT, Chandaratna AN, et al. Changes in blood rheology in patients with stable angina pectoris as a result of coronary artery disease. Circulation 1987;76: $15-20$

14 Neumann F-J, Tillmanns H, Roebruck P, Zimmermann R, Haupt $H-M$, Kübler W. Haemorrheological abnormalities in unstable angina pectoris: a relation independent of risk factor profile and angiographic severity. Br Heart J 1989;62:421-8.

15 Neumeier D, Prellwitz W, Würzburg U, et al. Determination of creatine kinase isoenzyme MB activity using immunological inhibition of creatine kinase $M$ subunit activity. Clin Chim Acta 1976;73:445-52.

16 Harkness J. A new instrument for the measurement of plasma viscosity. Lancet 1963;ii:280-1.

17 Volger E, Schmid-Schönbein H, VonGosen J, Klose HJ, Kline KA. Microrheology and light transmission of blood. IV. The kinetics of artificial red cell aggregation induced by dextran. Pflügers Arch 1975;354:319-37.

18 Neumann FJ, Schmid-Schönbein H, Ohlenbusch $H$. Temperature-dependence of red cell aggregation. Pflügers Arch 1987;408:524-30.

19 Clauss A. Gerinnungsphysiologische Schnellmethode zur Bestimmung des Fibrinogens. Acta Haematol (Basel) Bestimmung des

20 Judkins MP. Selective coronary angiography: a percutaneous transfemoral technique. Radiology 1967;89:815-24.

21 Hoberg E, Schwarz F, Voggenreiter U, Kübler W. Holter monitoring before, during, and after percutaneous transluminal coronary angioplasty for the evaluation of high resolution trend recordings of leads $\mathrm{CM}_{5}$ and $\mathrm{CC}_{5}$ for $\mathrm{ST}$ segment analysis. Am J Cardiol 1987;60:796-800.

22 Katus HA, Yasuda T, Gold HK, et al. Diagnosis of acute myocardial infarction: detection of circulating cardiac myosin light chains. Am J Cardiol 1984;54:964-8.

23 Katus HA, Remppis A, Looser S, Hallermeier K, Scheffold $T$, Kübler W. Enzyme linked immunoassay of cardiac troponin $T$ for the detection of acute myocardial infarction in patients. J Moll Cell Cardiol 1989;21:1349-53.

24 Schmid-Schönbein $\mathrm{H}$. Microrheology of erythrocytes, blood viscosity and the distribution of blood in the microcirculation. In: Guyton AC, Cowley AW, eds. International Reviews in Physiology vol. 9. Cardiovascular physiology II. Baltimore: University Park Press, 1976:
$1-62$.

25 Kushner I. The phenomenon of the Ann N Y Acad Sci 1982;389:39-48. syndrome in vascular disease. Int J Microcirc Clin Exp 1984;3:11-9.

27 Dinarello CA. Interleukin 1 and the pathogenesis of the acute-phase response. N Engl J Med 1984;311:1413-8.

28 Berk BC, Weintraub WS, Alexander RW. Elevation of Creactive protein in "active" coronary artery disease. Am J reactive protein in "active" 